\title{
Mirror symmetry and self-duality equations
}

\author{
Werner Nahm \\ Physikalisches Institut der Universität Bonn \\ Nussallee 12 \\ D-53115 Bonn
}

Abstract: A central problem of mirror symmetry is to understand Calabi-Yau moduli spaces at large complex structure. The basic techniques for this problem have been developped by Griffiths, Deligne and Schmid. The moduli space for complex structures is mapped into Griffiths' classifying space for Hodge structures, and the image is approximated by a nilpotent orbit up to exponentially small terms. Schmid described the geometry of the nilpotent orbit by equations which later were found by the speaker in the context of magnetic monopoles. The nilpotent orbit case corresponds to the special case of a maximally degenerate spectral curve.

\section{KEYWORDS: liarge complex structure, moduli, conformal field theory!.}

\section{Boundaries of the M-theory mod- uli space}

There are two limits in which non-perturbative string theory (alias M-theory) is accessible to calculations, namely small spacetime curvature and small string coupling. In the first case, in particular for strings on the product of a flat spacetime with a compact internal space of large radius, one obtains in the limit a conventional quantum field theory on an Einstein spacetime. The behaviour at small string coupling, on the other hand, is described by a superconformal theory on the string worldsheet. If the present indications of a low Higgs mass will hold true, one even can hope for low energy supersymmetry and consequently for extended supersymmetry in the worldsheet theory. Together with T- and S-duality, the two limits might give sufficient control over the boundaries of the (hypothetical) M-theory moduli space to get a handle on more realistic situations.

At present, even the understanding of the boundaries is far from complete. Even in the favorable case of extended supersymmetry, the conformal field theories are only solvable for special parameters, and there is still much to be learned from the case where the two limits are performed together. But at least a complete understanding of the geometry and metric of their moduli spaces seems feasible. This means that it will be much easier to classify the theories then to describe them in detail. Moreover, for the special physical quantities described by meromorphic functions the description of the moduli space takes us very close to an explicit computation, since for a conventional vacuum one expects compactifiable spaces.

\section{Classical and quantum moduli spaces}

The moduli space $M$ of a conformal theory with extended supersymmetry is closely related to certain moduli spaces of classical geometrical structures. In the limiting case when both the string coupling and the spacetime curvature tend to zero, it is clear that the conformal theory is given by a non-linear sigma model on a Calabi-Yau manifold $X$, such that $M$ can be projected to the moduli space $M(X)$ of complex structures of the underlying differentiable manifold. For IIA theories with (exactly) $N=(2,2)$ supersymmetry, in particular for central charges $c=3$ and $c=9$, one also obtains a projection to the moduli space of the mirror manifold $X^{\prime}$, and $M=$ $M(X) \times M\left(X^{\prime}\right)$, at least locally. The spaces $M(X), M\left(X^{\prime}\right)$ have complex structures of their own. 
For $c=6$ one does not find such a factorization. Indeed, mirror symmetry is obtained from the non-trivial automorphism of a $U(1)$ subgroup of the supersymmetry. For $c=6$, the supersymmetry gets extended to $N=(4,4)$, the $U(1)$ group is embedded in $S U(2)$ and all automorphisms are inner. Thus the moduli space is no longer described by the product of two moduli spaces of classical geometry. Nevertheless, the relation to classical structures is still close, as we shall see.

The classical moduli spaces have been studied extensively by mathematicians, and many results still have to be assimilated by physicists. On the other hand, the physics perspective opened new directions for mathematics, too, with many new directions to explore. The present talk will hint at some issues which may deserve more attention.

Omitting some subtleties, the classical moduli spaces are described as follows. Consider a manifold or algebraic variety $X$ of complex dimension $D=c / 3$ and its cohomology group $H^{*}(X, R)$. This is a real vector space with a sublattice $H^{*}(X, Z)$ and a wedge product. The projection of the latter to the generator of $H^{D}(X, R)$ yields groups $O\left(H^{\text {even }}(X, R)\right)$ and $S p\left(H^{o d d}(X, R)\right)$. The position of every natural subspace of $H^{*}(X, Z)$ yields continuous parameters, which can be described by positions in some coset of those groups.

Again, the case of complex dimension $D=2$ is somewhat special. Here Ricci flatness of the metric implies that $X$ is a torus or a $K 3$ variety. The torus case is easy, so let us consider $X=K 3$. In this case $H^{\text {odd }}$ vanishes, and all the interesting classical structure is contained in $H^{2}(X, R)$, which has 22 dimensions. Under the wedge product it has signature $(3,19)$. Moreover, on $H^{2}(X, R)$ the metric induces an action of the Hodge star operator. One is in the middle dimension, such that this automorphism of $H^{2}(X, R)$ is independent of the scale of the metric. Its square is 1 , such that $H^{2}(X, R)$ decomposes into its eigenspaces $\mathrm{H}^{+}$and $\mathrm{H}^{-}$on which the Hodge operator has eigenvalues +1 and -1 , resp. Locally, this yields a map from the moduli space of Ricci flat $K 3$ metrices to $S O\left(H^{2}(X, R)\right) /\left(S O\left(H^{+}\right) \times\right.$ $\left.S O\left(H^{-}\right)\right)$. It turns out that $H^{+}$has dimension 3 and is positive definite, whereas $H^{-}$is negative definite and has dimension 19.

Together with the integral sublattice, this decomposition is sufficient to classify the Ricci flat metrics. If one fixes the lattice, the Grassmannian of subspaces of dimension 3 has the form

$$
O(3,19) / O(3) \times O(19)
$$

and dimension 57. Actually one should be more careful about the connected components of the orthogonal groups [i]1].

If one takes into account the lattice automorphism group $\Gamma(3,19)$ the moduli space becomes

$$
\Gamma(3,19) \backslash O(3,19) / O(3) \times O(19) .
$$

This structure is typical, a non-compact Lie group modulo a maximal compact subgroup and an arithmetic subgroup.

In the string moduli space, the scale of the metric becomes important due to quantum corrections. Moreover, one has 22 B-field components, such that the complete moduli space has 80 dimensions. Roughly speaking, the moduli space takes the form

$$
\Gamma(4,20) \backslash O(4,20) / O(4) \times O(20) .
$$

Its points can be interpreted as specifying the relative position of a positive definite four-dimensional subspace and the integral lattice $H^{*}(X, Z)$ within the 24-dimensional space $H^{*}(X, R)$. This moduli space no longer has any symmetries, such that any conformal field theory which can be placed in it has a unique location. Finding this location is interesting, since one may be able to interpolate between two theories or otherwise explore the space of the corresponding string theories.

One example is given by the $Z_{2}$ orbifolds of four dimensional torus theories. The moduli space of the torus theories has the form

$$
\Gamma(4,4) \backslash O(4,4) / O(4) \times O(4)
$$

(Narain) and the $Z_{2}$ orbifolding procedure should yield an embedding. The torus moduli space corresponds to a sublattice of an eight dimensional space (the charge lattice of the theory), and a subspace which corresponds to the splitting of charges into left and right components.

On first sight, one might want to embed this space into the K3 moduli space by the direct sum 
with a standard 16 dimensional space, but this turns out to be wrong. When one takes parity into account, the action on the Narain and K3 spaces is incompatible with such a simple procedure. Instead, one first has to use $O(4,4)$ triality, which works analogously to the well known $O(8)$ triality. There is a two-fold ambiguity, which corresponds to the fact that generic points of the Narain moduli space correspond to two points of the K3 space, corresponding to two $N=2$ subalgebras of the $N=4$ supersymmetry. Moreover, the eight dimensional geometry has to be rescaled by a factor of two. A direct sum with a standard 16 dimensional space occurs, but this only yields a sublattice of finite index of the K3 lattice.

Similar results apply to other torus orbifolds, see [1] for $Z_{4}$ and [2] for $Z_{3}$ and $Z_{6}$. Finding the location of Gepner points of the K3 moduli space needs different techniques, but is possible, too.

\section{Schmid's work on large complex structures}

Now let us consider $c=9$, corresponding to strings on three dimensional Calabi-Yau varieties, which for simplicity we take to be simply connected. Their classical moduli space is given by the Hodge decomposition of the complexification of $H^{3}(X, R)$. More precisely, $H^{3}(X, R)$ decomposes into a two dimensional real vector space given by the real part of $H^{0,3} \oplus H^{3,0}$ and a complementary $H^{1,2} \oplus H^{2,1}$. On both of them, the Hodge decomposition yields a complex structure. Locally, this yields an embedding of the moduli space into $S p\left(H^{3}(X, R)\right) /\left(U(1) \times U\left(h^{1,2}\right)\right)$, where $h^{1,2}$ is the dimension of $H^{1,2}$. Globally, one still has to divide by the action of $S p\left(H^{3}(X, Z)\right.$.

Bryant and Griffiths have shown that one already obtains an embedding by considering the real part of $H^{0,3} \oplus H^{3,0}$, which gives an embedding into $S p\left(H^{3}(X, R)\right) /\left(U(1) \times S p\left(h^{1,2}\right)\right)$ [3i] The latter space has a complex structure given by the $U(1)$ action on its tangent space. Its complex dimension is $2 h^{1,2}+1$. The analogous space for $c=3$ is $S p(2) / U(1)$, which is just the complex upper half plane. The embedding of $M(X)$ is compatible with the complex structures.
The complex structure of the space $S p\left(H^{3}(X, R)\right) /\left(U(1) \times S p\left(h^{1,2}\right)\right)$ can be made manifest by including it in $S p\left(H^{3}(X, R) / B\right.$, where $B$ is a suitable Borel subgroup. In a suitable basis, $B$ is described by lower triangular matrices. In this basis, the grading of $g l(N)$ induced by $d\left(e_{i j}\right)=j-i$ for the elementary matrices $e_{i j}$ descends to a corresponding grading of $s p\left(H^{3}(X, R)\right.$. The grading counts the matrix diagonals with integers which are negative below the main diagonal and positive above.

Under mirror symmetry, rescaling the metric of $X$ corresponds to a large complex structure limit of the mirror $X^{\prime}$. In this limit the complex structure degenerates. In the following let us consider a classical type of moduli spaces with one complex parameter $q$, which for $q=0$ describes a degenerating complex structure (changing the parameter by $q^{\prime}=q+O\left(q^{2}\right)$ affects the calculation of non-perturbative effects but here it will not matter). In a well-known paper [4in], Schmid has proven two important theorems about this situation.

The first one is the nilpotent orbit theorem. It states that for some nilpotent generator $N$ of $S p\left(H^{3}(X, R)\right)$, the map from the $q$-plane to the moduli space can be approximated up to exponentially small terms by $q \mapsto \exp (N z) a$, where $q=\exp (2 \pi i z)$ and $a$ is a point in $S p\left(H^{3}(X, Z) / B\right.$. For consistency, $z$ and $z+1$ must yield the same point in the moduli space, such that $\exp (N)$ has to belong to $S p\left(H^{3}(X, Z)\right)$.

For the case of the mirror of the quintics

$$
\prod_{i=1}^{5} x_{i}=q \sum_{i=1}^{5} x_{i}^{5}
$$

which is given by the same equation modulo $Z_{5}$ actions, one easily obtains the values of $N$ and

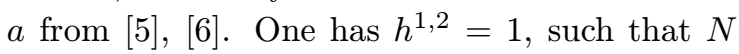
is a $4 \times 4$ matrix acting on a three-dimensional projective space, namely

$$
N=\left(\begin{array}{cccc}
10 & 11 / 2 & -5 & 0 \\
0 & 0 & 0 & 0 \\
20 & 10 & -10 & 0 \\
10 & -25 / 6 & -11 / 2 & 0
\end{array}\right)
$$

For the transpose of $a$ one finds.

$$
a^{t}=\left(-25 / 12,1,-25 / 6,25 i \zeta(3) \pi^{-3}\right)
$$


The transcendental entry in $a$ is remarkable, since everything else is given by rational numbers. Under mirror symmetry it is related to a loop integral, as discussed in $[\overline{\hat{n}}]$. The later mathematical reception of this paper did not comment on this number. One possible reason is that in the physics literature it occurs as constant term of the prepotential, a quantity with no clear mathematical meaning. As we have seen, it is implicit in the old mathematical literature, however. As a guess, it might be related to Deligne's work on mixed Hodge structures and Tate motives, which unfortunately remains largely unpublished.

The second theorem of Schmid is the $S L_{2}$ orbit theorem. It yields a more precise description of the orbit $q \mapsto \exp (N z) \circ a$, where we now include the real part of $H^{1,2} \oplus H^{2,1}$ in the description. Schmid shows that one can write

$$
\exp (N z) a=h(z) a
$$

where $h(z)^{-1} h^{\prime}(z)$ only has components of degrees 1 and -1 in the grading of $\operatorname{sp}\left(H^{3}(X, R)\right.$ introduced above. He changes variables to $z=i y$ and considers real $y$. He introduces the linear $\operatorname{map} \theta=(-)^{d}$ which acts as -1 on elements of odd degree of $s p\left(H^{3}(X, R)\right.$ and as +1 on elements of even degree. With

$$
\begin{aligned}
& A(z)=-2 h(y)^{-1} h^{\prime}(y) \\
& F(y)=h(y)^{-1} N h(y) \\
& E(y)=-\theta F(y)
\end{aligned}
$$

he shows that

$$
\begin{aligned}
2 E^{\prime}(y) & =-[A(y) E(y)] \\
2 F^{\prime}(y) & =[A(y) F(y)] \\
A^{\prime}(y) & =-[E(y) F(y)]
\end{aligned}
$$

Modulo a base change of $\operatorname{sl}(2)$ these are the same equations which were found later in the de-

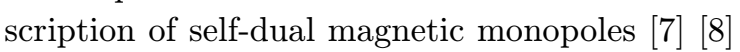
[ירוn].

When one conjugates by $h$, one obtains differential equations for the matrices $h^{\prime}(y) h(y)^{-1}$ and $h(y) E(y) h(y)^{-1}$. These equations can be solved in terms of Riemannian theta functions and a linear flow on the Jacobian of a spectral curve. In Schmid's case the spectral curve is maximally degenerate, since for $y \rightarrow \infty$ all matrices converge to zero. This means that the entries of $h^{\prime}(y) h(y)^{-1}$ and $h(y) E(y) h(y)^{-1}$ are rational functions of $y$, a fact proved by Schmid in a different way.

Since a lot of experience has beeen gained in the solution of the monopole equations, it may be interesting to return to Schmid's work from this perspective.

Schmid's construction shows that $N$ has a natural interpretation as the raising generator of an $s l_{2}$ Lie algebra. Under mirror symmetry this $s l_{2}$ given by the limit of large complex structure corresponds to the $s l_{2}$ generated by the Kähler operator and its adjoint. The analogy between the two Lie algebras is stressed on page 249 of [4] a remarkably early premonition of mirror symmetry, and another instance of astonishing paralellism in the development of mathematics and physics.

\section{References}

[1] W. Nahm, K. Wendland, A Hiker's Guide to K3, hep-th/9912067, to appear in CMP

[2] K. Wendland, Consistency of Orbifold Conformal Field Theories on K3, hep-th/0010281

[3] R. Bryant, P. Griffiths, Some observations on the infinitesimal period relations for regular threefolds with trivial canonical bundle, in: Arithmetic and Geometry, Vol. 2, Birkhäuser 1983

[4] W. Schmid, Inventiones math. 22 (1973) 211319

[5] P. Candelas et al., Nuclear Phys. B359 (1991) 21-74

[6] D.R. Morrison, J.AMS 6 (1993) 223-241

[7] W. Nahm, The construction of all self-dual multi-monopoles by the ADHM method, in: Monopoles in quantum field theory, Craigie et al. eds., World Scientific, Singapore 1982

[8] N.J. Hitchin, CMP 89 (1983) 145-190

[9] N. Ercolani, A. Sinha, CMP 125 (1989) 385416 